\title{
A High Aspect-Ratio Polysilicon Vibrating Ring Gyroscope
}

\author{
Farrokh Ayazi*, Hsiao H. Chen, Fatih Kocer, Guohong He, and Khalil Najafi \\ Center for Integrated MicroSystems \\ University of Michigan, 1301 Beal Avenue, Ann Arbor, MI 48109-2122 \\ Email: najafi@umich.edu, Tel: (734) 763-6650,Fax: (734) 763-9324 \\ *Currently with: Georgia Institute of Technology, Atlanta, GA 30332-0250
}

\begin{abstract}
This paper presents the fabrication and testing of a high aspect-ratio $80 \mu \mathrm{m}$ tall polysilicon ring gyroscope (PRG) fabricated using a new dry-release poly-silicon MEMS technology. This single-wafer technology is capable of producing electrically isolated vertical electrodes as tall as the main body polysilicon structure ( 50 to 100 's $\mu \mathrm{m}$ tall) with various size air-gaps ranging from sub-micron to tens of microns. An open-loop sensitivity of $200 \mu \mathrm{V} / \mathrm{deg} / \mathrm{sec}$ in a dynamic range of $\pm 250 \mathrm{deg} / \mathrm{sec}$ was measured under low vacuum level for a prototype device tested in hybrid format. The resolution for a prototype sensor with a quality factor (Q) of 1200 , drive amplitude of $0.15 \mu \mathrm{m}$, and sense node parasitic capacitance of $2 \mathrm{pF}$ was measured to be less than $1 \mathrm{deg} / \mathrm{sec}$ in $1 \mathrm{~Hz}$ bandwidth. Elimination of the parasitic capacitance and improvement in the quality factor of the ring structure will improve the resolution to $0.01 \mathrm{deg} / \mathrm{sec}$ in $1 \mathrm{~Hz}$ bandwidth. Three hundred microns long clamped-clamped beam resonators fabricated in this technology have shown measured quality factors as high as 85,000 in $1 \mathrm{~m}$ Torr yacuum.
\end{abstract}

Keywords: Gyroscope, high aspect-ratio, silicon micromachining, vibratory gyroscope, yaw rate sensor.

\section{INTRODUCTION}

High-performance microgyroscopes [1] are needed in many applications, including inertial navigation, control, and defense/avionics/space. The first nickel vibrating ring gyroscope was presented at the 1994 Hilton Head Workshop [2]. The vibrating ring gyroscope, shown in Fig. 1, consists of a ring, eight semicircular support springs, and drive, sense and control electrodes that are located around the structure. The ring is electrostatically vibrated into its first flexural mode with fixed amplitude. When the device is subjected to rotation around its normal axis, Coriolis force causes energy to be transferred from the first mode to the second flexural mode, which is located $45^{\circ}$ apart from the first mode, causing amplitude to build up proportionally in the latter mode; this build-up is capacitively monitored. This device provides a number of advantages, including excellent mode matching, high-resolution, low zero-rate output, and long-term stability. The technological requirements for improving gyroscope performance and the scaling limits of ring gyroscopes were previously presented at MEMS 98 conference [3]. This paper reports the fabrication and testing of a high aspect-ratio (20:1), $80 \mu \mathrm{m}$ tall, $1.1 \mathrm{~mm}$ in diameter polysilicon ring gyroscope (PRG) fabricated using a new dry-release poly-silicon MEMS technology [4]. This all silicon single-wafer technology is capable of producing electrically isolated vertical electrodes as tall as the main body structure ( 50 to 100 's $\mu \mathrm{m}$ tall) with various size air gaps ranging from sub-micron to tens of microns. It provides all the features required for eventual realization of "inertial-grade" micromachined gyroscopes and other MEMS devices.

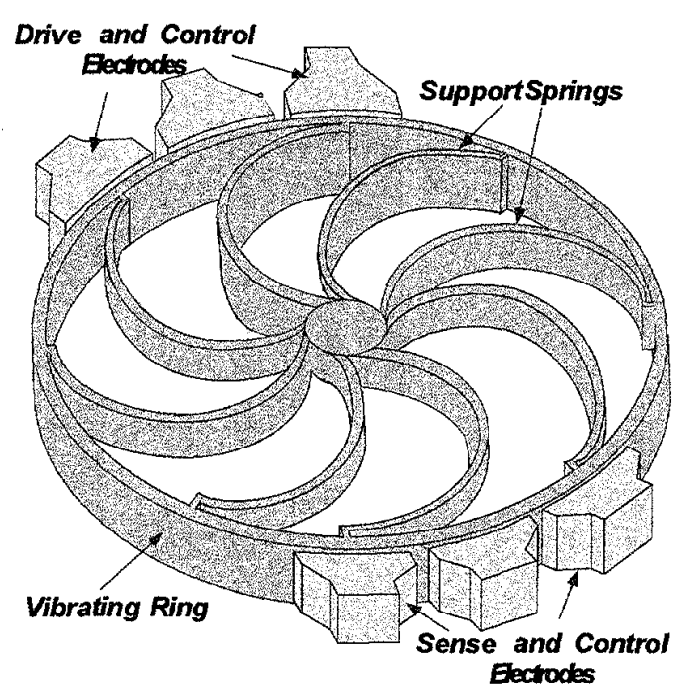

Figure 1: Structure of a vibrating ring gyroscope.

\section{FABRICATION TECHNOLOGY}

Figure 2 shows the fabrication process flow for the high aspect-ratio dry-release poly-silicon technology. First, a thin layer of LPCVD silicon nitride is deposited and patterned to serve as an isolation dielectric layer underneath the electrode bonding pads. Deep trenches with straight sidewalls $\left(90^{\circ} \pm 1^{\circ}\right)$ are then dry etched into a low-resistivity silicon substrate using the STS Silicon Deep Reactive Ion Etcher (DRIE). Trenches with smooth and straight sidewalls are needed to eliminate void formation in the polysilicon beams which will be formed by back-filling these trenches. Mechanical structures are created by refilling trenches with polysilicon deposited over a sacrificial oxide layer. The structural polysilicon layer has to be doped to lower its electrical resistance. Doping with boron is preferred over phosphorous due to the fact that the etch rate of boron-doped poly is much less than the etch rate of phosphorous-doped poly in the $\mathrm{HF}: \mathrm{H}_{2} \mathrm{O}(1: 1)$ solution, leaving the poly beams intact after relatively long HF release step.

Silicon sense electrodes as tall as the ring structure are released from the substrate using a dry directional/isotropic $S F_{6}$ silicon etch performed in an STS etcher. This dry release step consists of a deep, directional etch (depth of the trenches $+20 \mu \mathrm{m}$ ) followed by an isotropic $\mathrm{SF}_{6}$ silicon etch. The sacrificial oxide layers are then etched away in $\mathrm{HF}: \mathrm{H}_{2} \mathrm{O}(1: 1)$ solution to create submicron capacitive air gaps between the sense-electrodes and the ring structure.

This technology has been employed to fabricate a number of thick polysilicon vibrating shell gyroscopes [4] with silicon electrodes as tall as the shell structures. Figure 3 shows the SEM view of an $80 \mu \mathrm{m}$ tall, polysilicon ring gyroscope. The ring is 
$1.1 \mathrm{~mm}$ in diameter and the diameter of the support post is $120 \mu \mathrm{m}$. The width of the ring and support springs is $4 \mu \mathrm{m}$. Sixteen electrodes are evenly located around the structure; they are approximately $60 \mu \mathrm{m}$ tall and $150 \mu \mathrm{m}$ long and are separated from the ring by a $1.4 \mu \mathrm{m}$ capacitive air-gap. Figure 4 shows close-up of a silicon electrode as tall as the main body structure separated from $80 \mu \mathrm{m}$ thick polysilicon ring by $1.4 \mu \mathrm{m}$ air-gap created through sacrificial oxide etching. The silicon is anchored on top to the supporting polysilicon layer, which is in turn anchored through the isolating nitride layer to the substrate. Vertical polysilicon stiffeners (trench-refilled) are also incorporated in the structure of the electrodes to improve rigidity.

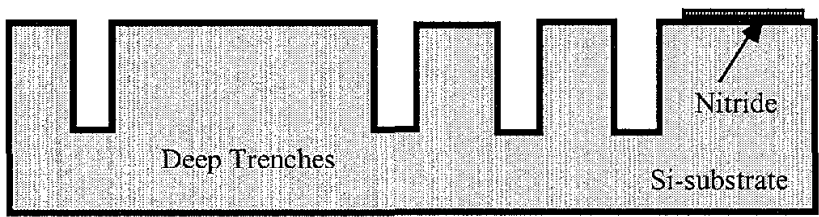

a) Deposit and pattern the isolating nitride layer;

b) dry etch deep trenches to define the main body structure.

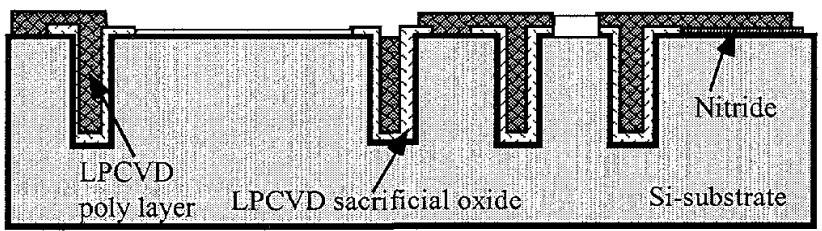

c) Deposit LPCVD sacrificial oxide and dope surface of the oxide; d) refill trenches with LPCVD polysilicon; e) etch back poly; f) pattern oxide; g) deposit, dope and pattern poly.

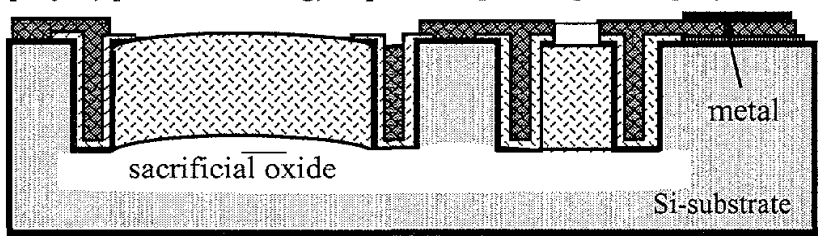

h) Deposit and pattern $\mathrm{Cr} / \mathrm{Au}$;

i) $\mathrm{SF}_{6}$ dry directional etch + undercut and release electrodes;

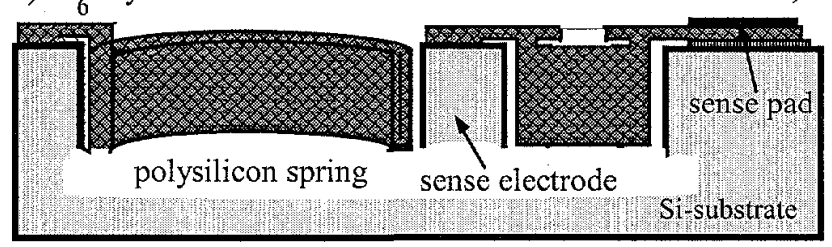

j) Etch the sacrificial oxide and completely release structures.

Figure 2: Fabrication process flow for the six-mask, high aspectratio dry-release poly-silicon MEMS technology.

Size of the air-gaps in this technology is not limited to submicron levels. Larger air-gaps can be realized with polysilicon trench-refilled electrodes. Larger air-gaps are defined by the distance between two adjacent trenches that form the parallel plates of a capacitor. The silicon between these trenches is then directionally etched in the STS machine during the deep release step. If the spacing is so small that etch openings cannot be placed between adjacent trenches, then the silicon between the two trenches is protected with resist on the top but undercut at the bottom and the sides during the deep release step. This narrow piece of silicon which is held by the oxide on the sidewall of trenches will then fall into the $\mathrm{HF}: \mathrm{H}_{2} \mathrm{O}$ solution during the sacrificial oxide etch. Figure 5 shows the SEM view of wide capacitive gaps $(10-20 \mu \mathrm{m})$ with polysilicon electrodes fabricated using this technology.

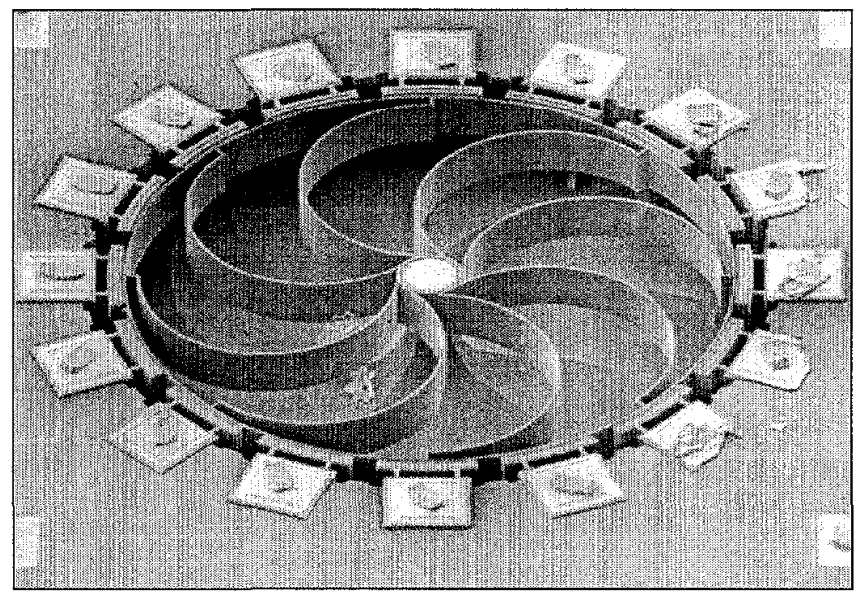

Figure 3: $\mathrm{SEM}$ view of a prototype $1.7 \mathrm{~mm} \times 1.7 \mathrm{~mm}$ polysilicon ring gyroscope fabricated through this technology. The dry-release process was entirely carried in the STS machine.

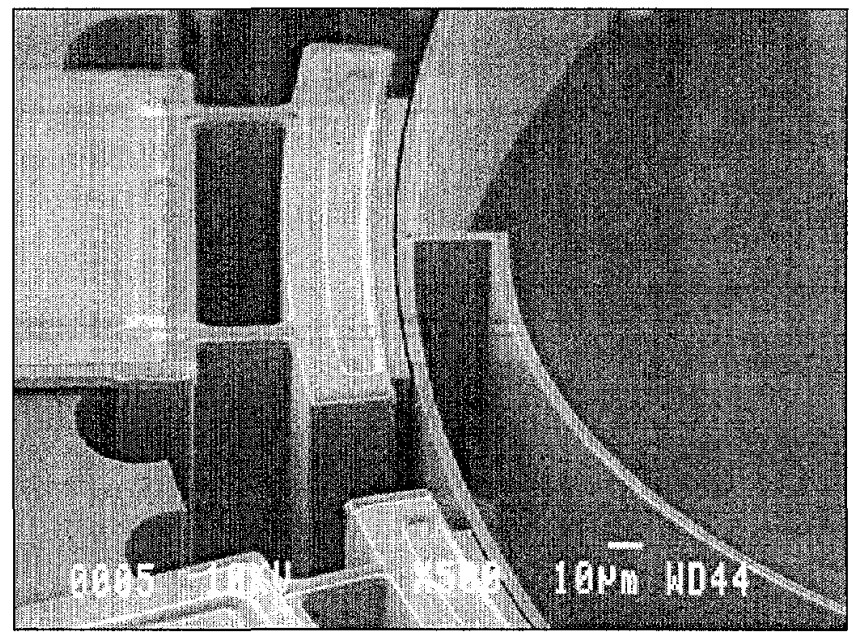

Figure 4: Close-up of a released sense electrode separated by $1.4 \mu \mathrm{m}$ air-gap from the vibrating ring. Each sense electrode is $60 \mu \mathrm{m}$ tall. Ring and springs are $4 \mu \mathrm{m}$ wide and $80 \mu \mathrm{m}$ tall.

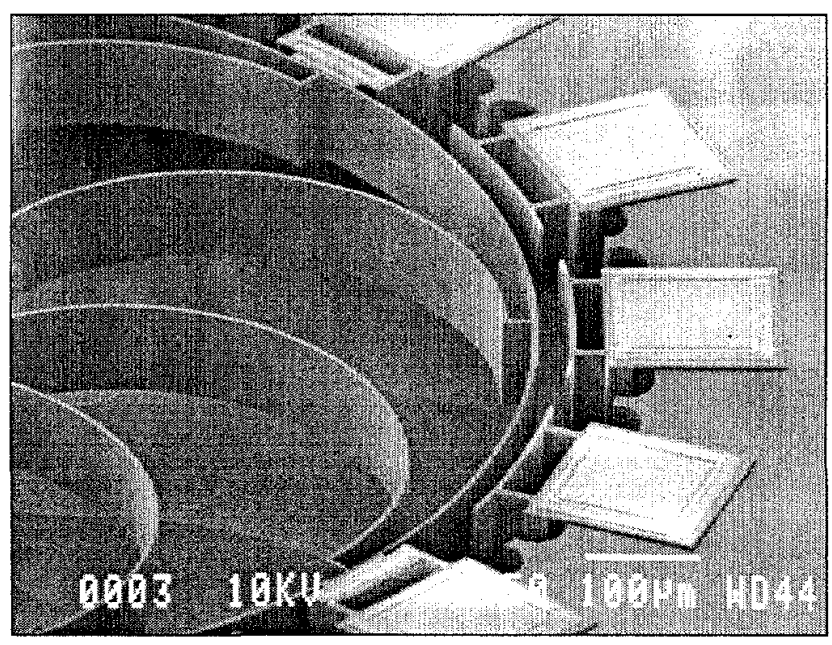

Figure 5: Various size air gaps from sub-micron to tens of micron. 
This technology provides several important features required for high-performance microgyroscopes. First, the height of the polysilicon main body structure "and the electrodes" can be increased to 100 's $\mu \mathrm{m}$ using DRIE. Second, since the body to sense-electrode gap is defined by the thickness of the sacrificial layer, it can be reduced to sub-micron level; these two factors together will significantly increase the sense capacitance and hence the sensitivity. Third, the ring to drive-electrode spacing can be made arbitrarily larger than the ring to sense-electrode spacing to achieve larger drive amplitude and in turn, reduce the noise floor. Fourth, improved long-term stability and temperature sensitivity is obtained due to the all-silicon feature of this technology.

\section{TEST RESULTS}

An $80 \mu \mathrm{m}$ tall prototype polysilicon ring gyroscope was tested open loop under vacuum. The sensor chip was connected in hybrid format to an NMOS source-follower buffer chip, as shown in Fig. 6, to measure ring vibrations. The low-impedance output signal of this buffer chip was then amplified off-chip using discrete amplifiers. Drive and control circuitry needed for tuning and open or closed loop operation of the ring gyroscope were implemented off chip using discrete components.

Electronic tuning is an attractive feature of the ring gyroscope. Any frequency mismatch between the sense and drive modes is electronically compensated using tuning electrodes around the structure. By matching the drive and sense mode frequencies, sensitivity is amplified by the $\mathrm{Q}$ of the structure. The frequency of the flexural mode of a prototype device is measured to be $\sim 28.3 \mathrm{kHz}$ (with $7 \mathrm{~V}$ bias applied to the ring) which agrees well with calculation and ANSYS simulation results. As shown in Fig. 7, drive and sense mode frequencies of the ring gyro were $63 \mathrm{~Hz}$ apart due to imperfections. The balancing voltages required to null this $63 \mathrm{~Hz}$ of frequency split between the modes were only $\pm 0.9 \mathrm{~V}$. After balancing, the two peaks merge together and the sense and drive mode frequencies become equal. We have also been able to balance ring structures with up to $1 \mathrm{kHz}$ of frequency split $\left(\mathrm{f}_{0}=28 \mathrm{kHz}\right.$ ) by applying less than 6 Volts tuning voltages to the balancing electrodes. Small capacitive gaps $(1.4 \mu \mathrm{m})$ have indeed shifted the tuning voltages down to CMOS acceptable levels, increasing the tuning capability.

The measured $\mathrm{Q}$ of $1.1 \mathrm{~mm}, 80 \mu \mathrm{m}$-thick ring structure was $\approx 1000$-2000. This is much smaller than anticipated due to excessive

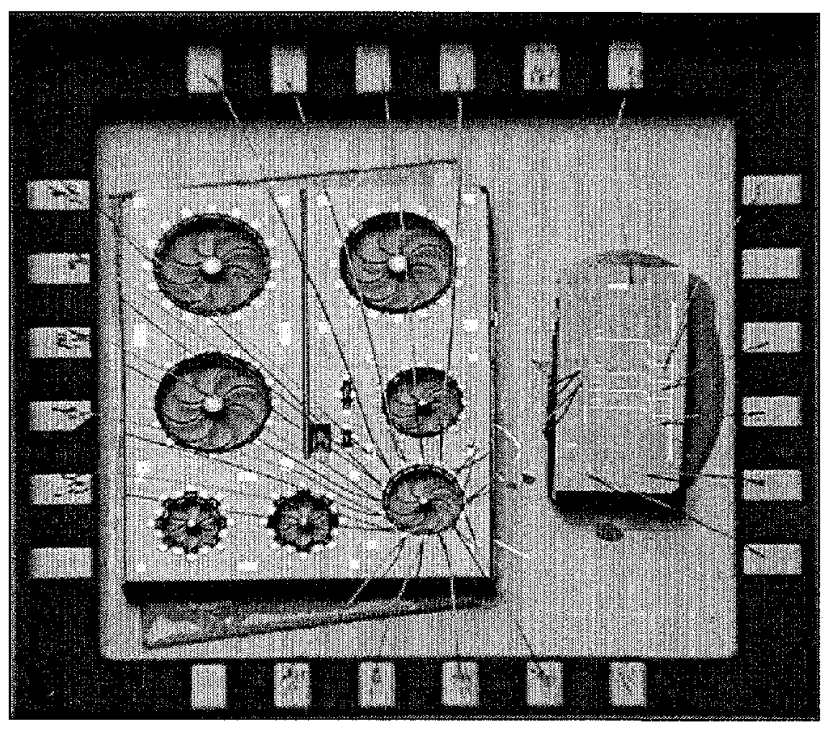

Figure 6: Hybrid attachment of the sensor chip to the buffer chip.

anchor and bulk losses (voids inside the poly beams). Modified devices with solid post design are expected to have higher $Q$ in the range of 10,000-20,000 and hence higher sensitivity. $80 \mu \mathrm{m}$ tall, 300 um long clamped-clamped beam micromechanical resonators fabricated in this technology have shown quality factors as high as 85,000 in $1 \mathrm{~m}$ Torr vacuum [4].

Figure 8 shows the response of a prototype ring gyroscope to a $5 \mathrm{~Hz}$ sinusoidal input rotation rate with peak-to-peak amplitude of $120 \mathrm{deg} / \mathrm{sec}$. Figure 9(a) shows the response of a prototype device to input rotation rates up to $\pm 250 \mathrm{deg} / \mathrm{sec}$ in a $5 \mathrm{~Hz}$ bandwidth. It should be noted that this measurement was carried out under poor vacuum level and the quality factor was about 250 which is much less than the actual $Q$ of the ring structure under high vacuum. This drop in the quality factor reduces the open loop output signal by a factor of 5-8. Also, the output signal has been further reduced due to large parasitic capacitance in the output sense node. The parasitic capacitance associated with the hybrid connection $\left(\mathrm{C}_{\mathrm{inp}}\right.$ of the buffer + capacitance of bonding pads and wirebonds) have been measured to be around $2 \mathrm{pF}$ total. This large capacitance has degraded the output signal by an additional factor of 5 . The sensitivity of a prototype device under poor vacuum $(Q \approx 250)$ and large parasitics $(\mathrm{C} \approx 2 \mathrm{pF})$ was measured to be $200 \mu \mathrm{V} / \mathrm{deg} / \mathrm{sec}$.

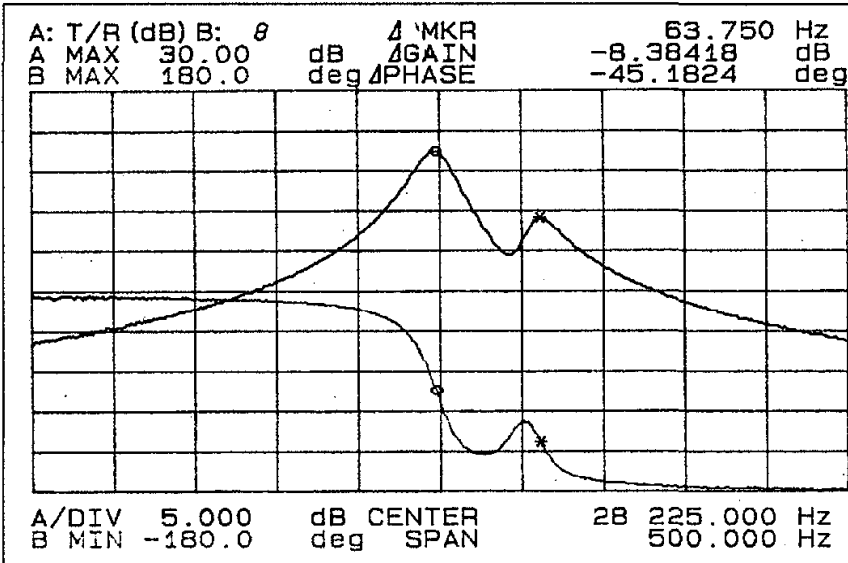

(a)

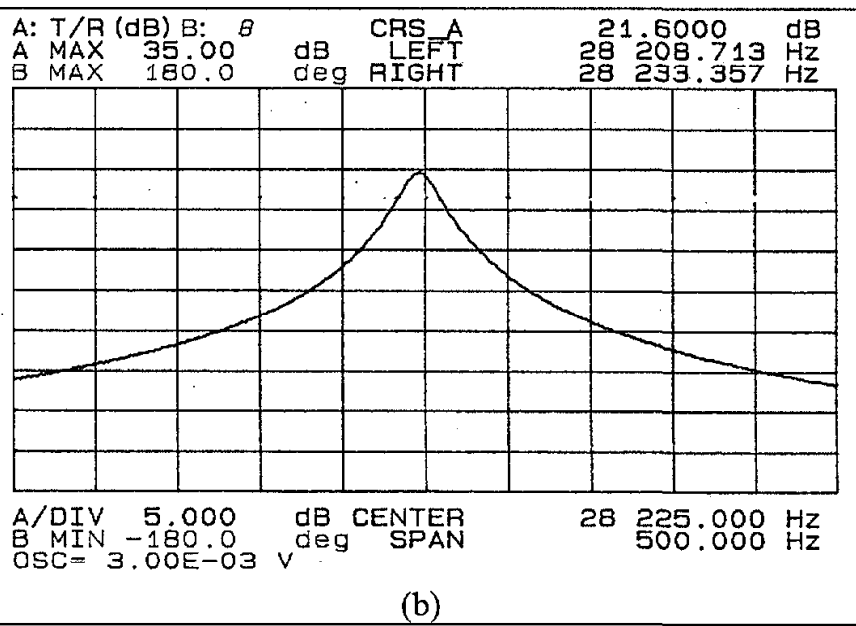

(b)

Figure 7: Elcctronic balancing of the ring gyroscope. (a) Before balancing: Two flcxural resonant peaks of a prototypc ring gyroscope have different frequencies $\left(\Delta \mathrm{f}=63 \mathrm{~Hz}\right.$ for $\mathrm{f}_{0}=28 \mathrm{kHz}$ ). (b) After balancing: two peaks merge together and the frequencies become equal. 


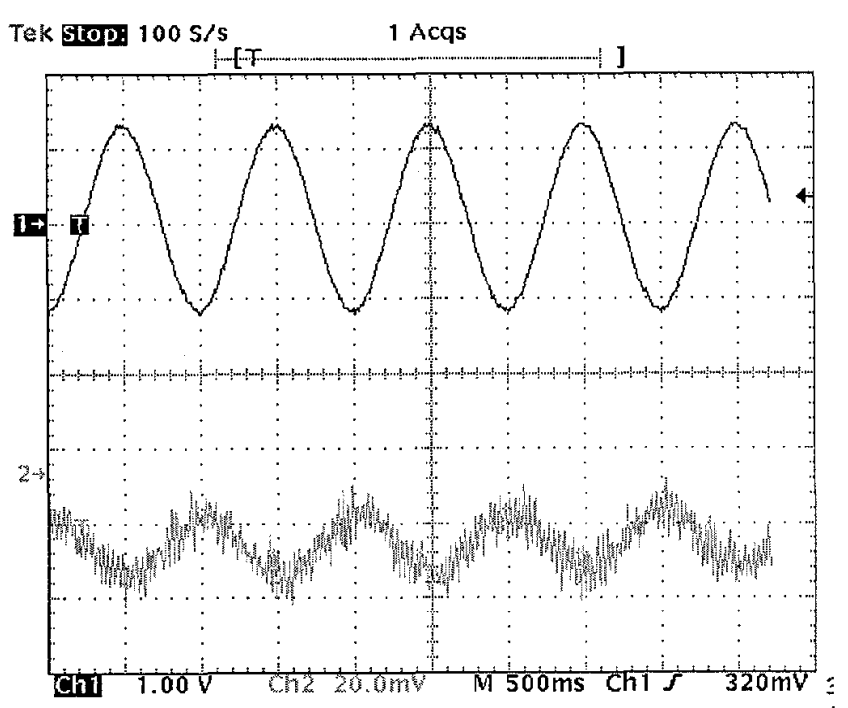

Figure 8: Response of a prototype single ring gyroscope to a $5 \mathrm{~Hz}$ sinusoidal rate with a peak-to-peak amplitude of $120 \mathrm{deg} / \mathrm{sec}$.

Another set of measurements was taken under improved vacuum levels. The $Q$ was measured to be $\approx 1200$ after balancing. Figure $9(\mathrm{~b})$ shows the response of the sensor to rotation rates in the range of $-20 \mathrm{deg} / \mathrm{sec}$ to $20 \mathrm{deg} / \mathrm{sec}$. The resolution was measured to $\mathrm{be} \approx 1 \mathrm{deg} / \mathrm{sec}$. This resolution for a $\mathrm{Q}$ of 1200 and parasitic capacitances of $2 \mathrm{pF}$ is in agreement with theory. The resolution of the sensor is currently limited by the noise from the circuitry. Elimination of parasitic capacitances and improvement in $Q$ will reduce the resolution to $0.01 \mathrm{deg} / \mathrm{sec}$ in $1 \mathrm{~Hz}$ bandwidth, which is the Brownian noise floor for the single ring polysilicon gyroscope with a drive amplitude of $0.15 \mu \mathrm{m}$ (Table 1). By increasing the drive amplitude to $1 \mu \mathrm{m}$ in an asymmetric polysilicon ring gyroscope design, a minimum detectable signal of $5 \times 10^{-3} \mathrm{deg} / \mathrm{sec}$ $(18 \mathrm{deg} / \mathrm{h})$ in a $10 \mathrm{~Hz}$ bandwidth can be achieved.

\section{CONCLUSIONS}

This paper presents the fabrication and testing of a high aspect-ratio $80 \mu \mathrm{m}$ tall polysilicon ring gyroscope fabricated using a new dry-release poly-silicon MEMS technology. An open-loop sensitivity of $200 \mu \mathrm{V} / \mathrm{deg} / \mathrm{scc}$ in a dynamic range of $\pm 250 \mathrm{deg} / \mathrm{sec}$ was measured under low vacuum level for a prototype device tested in hybrid format. The resolution for a prototype sensor with a quality factor $(Q)$ of 1200 , drive amplitude of $0.15 \mu \mathrm{m}$, and sense-

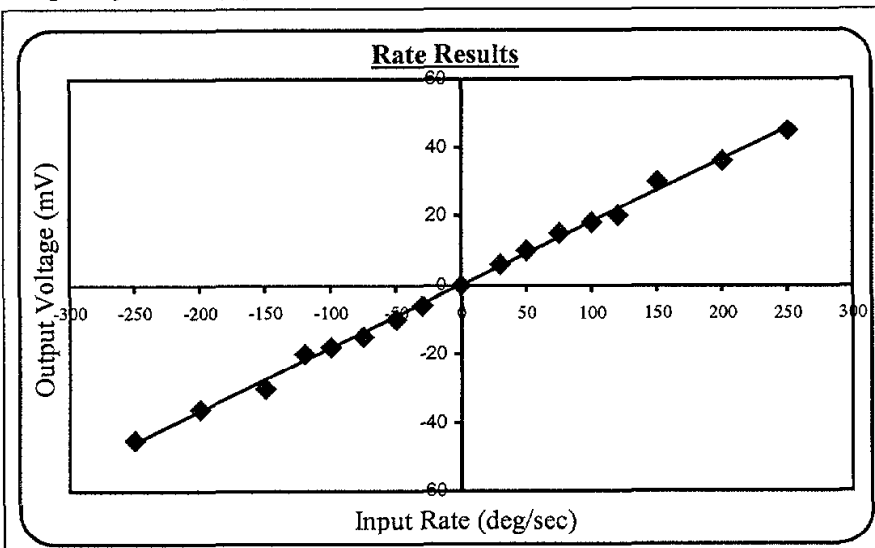

(a) node parasitic capacitance of $2 \mathrm{pF}$ was measured to be less than 1 $\mathrm{deg} / \mathrm{sec}$ in $1 \mathrm{~Hz}$ bandwidth. Elimination of the parasitic capacitance and improvement in the quality factor of the ring structure will improve the resolution to $0.01 \mathrm{deg} / \mathrm{sec}$ in $1 \mathrm{~Hz}$ bandwidth.

Table 1: Specifications and calculated performance of the polysilicon vibrating ring gyroscope.

\begin{tabular}{|c|c|}
\hline Design Parameter & Target Value \\
\hline Material Quality Factor & 20,000 \\
\hline Ring Diameter & $1.1 \mathrm{~mm}$ \\
\hline Sense Electrode Gap Spacing & $1.4 \mu \mathrm{m}$ \\
\hline Ring and Springs Width & $4 \mu \mathrm{m}$ \\
\hline Height of the Ring Structure & $80 \mu \mathrm{m}$ \\
\hline Support Post Diameter & $120 \mu \mathrm{m}$ \\
\hline Height of Each Electrode & $60 \mu \mathrm{m}$ \\
\hline Resonant Frequency & $27.3 \mathrm{kHz}$ \\
\hline Interface Circuit Input Noise $\left(\mathrm{V}_{\mathrm{n}}\right)$ & $1 \mu \mathrm{V} /(\mathrm{Hz})^{0.5}$ \\
\hline Parasitic Capacitance $\left(\mathrm{C}_{\text {device }}+\mathrm{C}_{\mathrm{pad}}\right)$ & $500 \mathrm{fF}$ \\
\hline Polarization Voltage $\left(\mathrm{V}_{\mathrm{p}}\right)$ & $7 \mathrm{~V}$ \\
\hline Drive amplitude & $0.15 \mu \mathrm{m}$ \\
\hline Brownian Noise Floor & $0.01 \mathrm{deg} / \mathrm{sec} /(\mathrm{Hz})^{0.5}$ \\
\hline Min. Detectable Signal $(\mathbf{1 0 H z}$ BW) & $\mathbf{0 . 0 4} \mathrm{deg} / \mathrm{sec}$ \\
\hline
\end{tabular}

\section{ACKNOWLEDGMENTS}

This work is supported by the Defense Advanced Research Projects Agency (DARPA) under contract \# F30602-98-2-0231. The authors wish to thank Dr. Mike Putty and Mr. Larry Oberdier of Delphi Automotive for their help in testing.

\section{REFERENCES}

[1] N. Yazdi, F. Ayazi, and K. Najafi, "Micromachined Inertial Sensors," Invited paper, Proceedings of the IEEE, pp.1640-1659, Aug. 1998.

[2] M. W. Putty and K. Najafi, "A Micromachined Vibrating Ring Gyroscope", Digest, Solid-State Sensors and Actuators Workshop, Hilton Head, SC, June 1994, pp. 213-220.

[3] F. Ayazi and K. Najafi, "Design and Fabrication of a HighPerformance Polysilicon Vibrating Ring Gyroscope," in Proc. 1998 IEEE/ASME Micro Electro Mechanical Systems Workshop, Heidelberg, Germany, 1998, p. 621.

[4] F. Ayazi and K. Najafi, "High Aspect-Ratio Dry-Release Poly-Silicon MEMS Technology for Inertial Grade Microgyroscopes," in Proc. IEEE Position Location And Navigation Symposium (PLANS 2000), San Diego, CA, March 2000, pp. 304-308.

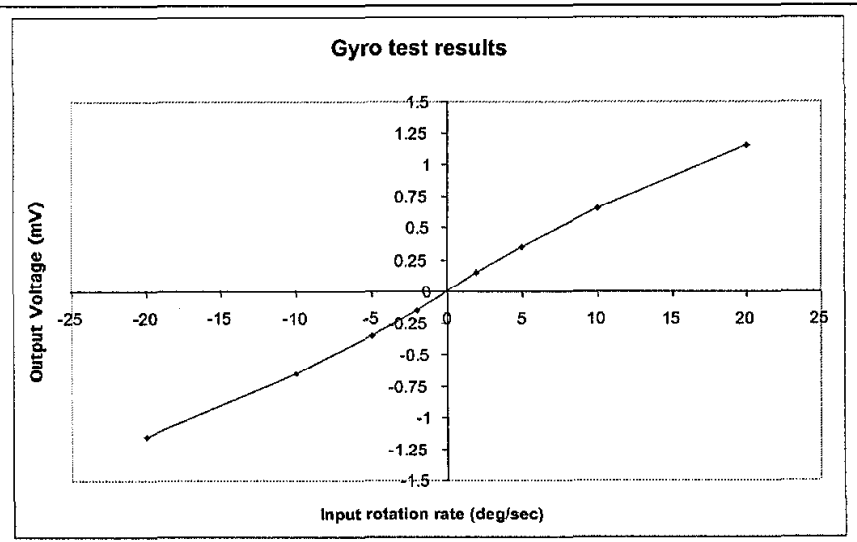

(b)

Figure 9: (a) Measured rate results obtained from a prototype ring gyroscope in a $5 \mathrm{~Hz} \mathrm{BW}$, range $= \pm 250 \%$ sec; (b) Measured rate results obtained from a prototype ring gyroscope in a $5 \mathrm{~Hz} \mathrm{BW}$, range $= \pm 25 \%$ sec. 